\title{
Prevalence and Risk Factors of Ascaris lumbricoides, Trichuris trichiura and Cryptosporidium Infections in Elementary School Children in Southwestern China: A School-Based Cross-Sectional Study
}

\author{
Dongjian Yang ${ }^{1,2,3,+}+{ }^{(0)}$, Ya Yang ${ }^{1,2,3,+}$, Yingjian Wang ${ }^{1,2,3}$, Yu Yang ${ }^{1,2,3}$, Shurong Dong ${ }^{1,2,3}$, \\ Yue Chen ${ }^{4}$, Qingwu Jiang ${ }^{1,2,3}$ and Yibiao Zhou ${ }^{1,2,3, *}$ \\ 1 Department of Epidemiology, School of Public Health, Fudan University, 138 Yi Xue Yuan Road, \\ Shanghai 200032, China; 17211020111@fudan.edu.cn (D.Y.); yayang16@fudan.edu.cn (Y.Y.); \\ 17211020008@fudan.edu.cn (Y.W.); 16211020071@fudan.edu.cn (Y.Y.); 17211020051@fudan.edu.cn (S.D.); \\ jiangqw@fudan.edu.cn (Q.J.) \\ 2 Key Laboratory of Public Health Safety, Fudan University, Ministry of Education, Shanghai 200032, China \\ 3 Center for Tropical Disease Research, Fudan University, Shanghai 200032, China \\ 4 School of Epidemiology and Public Health, Faculty of Medicine, University of Ottawa, Ottawa, \\ ON K1G 5Z3, Canada; Yue.Chen@uottawa.ca \\ * Correspondence: z_yibiao@hotmail.com or ybzhou@fudan.edu.cn \\ + These authors contributed equally to this work.
}

Received: 27 June 2018; Accepted: 20 August 2018; Published: 22 August 2018

\begin{abstract}
Background: Intestinal parasitic infections pose great public health challenges in school children in developing countries. The aim of this study was to assess the prevalence of A. lumbricoides, T. trichiura and Cryptosporidium among elementary school children in rural southwestern China. Methods: A school-based cross-sectional study involving 321 elementary school children was conducted in 2014 in the southwest of China. They were invited to provide a stool sample and interviewed about the sanitary situation and hygiene behavior. Stool specimens were examined for A. lumbricoides and T. trichiura using the Kato-Katz fecal thick-smear technique. The presence of Cryptosporidium was determined using a modified acid-fast staining method. Results: The prevalence of infection was 10.0\% (95\% CI: $6.9-13.8 \%$ ) for A. lumbricoides, $25.2 \%$ (95\% CI: 20.6-30.4\%) for T. trichiura and $2.4 \%$ for (95\% CI: 1.1-4.9\%) Cryptosporidium. The prevalence of co-infection was 3.7\% (95\% CI: 1.9-6.4\%) for A. lumbricoides / T. trichiura, 0.3\% (95\% CI: 0-1.7\%) for A. lumbricoides/Cryptosporidium and $0.9 \%$ (95\% CI: 0.2-2.7\%) for T. trichiura/Cryptosporidium. Children from households using well or river water were associated with a greater odds of $A$. lumbricoides infection (aOR $=2.61,95 \% \mathrm{CI}: 1.12-6.05)$. Having a household lavatory was associated with a lower odds of T. trichiura infection (aOR $=0.50,95 \%$ CI: 0.30-0.84). Children who had three meals at the school canteen on week days were at a lower risk of Cryptosporidium infection. The use of spring water as a water source was associated with lower odds of any intestinal infection $(\mathrm{aOR}=0.56$, 95\% CI: 0.35-0.91). Conclusions: Our study calls for an intervention program of school-based deworming combined with health education, hygiene promotion and provision of safe water and improved sanitation.
\end{abstract}

Keywords: A. lumbricoides; T. trichiura; Cryptosporidium; school children; China

\section{Introduction}

Infections with helminths (e.g., Ascaris lumbricoides, Trichuris trichiura and hookworm) and intestinal protozoa (e.g., Cryptosporidium and Giardia intestinalis) are global public health threats 
and are closely associated with poverty, unsafe water and inadequate sanitation and hygiene [1]. More than one billion people worldwide are infected with one or more species of soil-transmitted helminths (STH), of which over 267 million pre-school children and over 568 million school children are considered at risk of morbidity, especially in less developed countries [1-3]. STH infections fall within the grouping termed as neglected tropical diseases (NTDs), and nearly $70 \%$ of the global burden it causes occurs in Asia [4,5]. Infections can cause diarrhea, abdominal pain, malnutrition, physical and intellectual growth retardations [1]. Cryptosporidiosis is a major cause of diarrheal disease in developing and developed countries, and epidemiological studies have shown that Cryptosporidium is more prevalent in developing countries (5\% or higher) than in developed countries ( $3 \%$ or less) [6]. Cryptosporidium was identified to be the second only to rotavirus as a cause of moderate-to-severe diarrhea in children during the first two years of life [7]. Infection with Cryptosporidium can lead to self-limiting diarrhea in immunocompetent individuals but life-threatening and prolonged diarrhea in immunocompromised ones such as people infected with HIV [8]. Cryptosporidium infection is also associated with malnutrition and growth deficits in children. The types of organisms, risk factors and routes of transmission of the three infectious diseases are different, but their transmission is related to poor sanitation conditions and sanitary practices (Table 1) [9].

Historically, infections of $A$. lumbricoides and T. trichiura, along with other intestinal helminth parasites, have been a major public health problem in China. There have been two national surveys of parasitic diseases conducted in China. The prevalence of infection was $47.0 \%$ for A. lumbricoides and $18.8 \%$ for T. trichiura in the first national survey of parasitic diseases (1988-1992), which dropped dramatically to $12.7 \%$ and $6.1 \%$ at the second national survey (2001-2004) [10]. Although, the prevalence of STH infections continuously decreased according to national surveillance data, A. lumbricoides and T. trichiura infections remain high in several underdeveloped regions in China [11-14]. A review of South and Southeast Asia demonstrates that school children have higher infection risks of $A$. lumbricoides (25\%, 95\% CI: $16-31 \%)$ and T. trichiura (22\%, 95\% CI: $14-34 \%)$ than the general population [15]. However, Cryptosporidium infection had been largely neglected and few studies had been conducted in the Yi ethnicity in SW China. Similarly, there are few studies on the epidemiology of STH infections and risk factors for infection in primary school children. Studies of A. lumbricoides, T. trichiura and Cryptosporidium in children are relevant to the control of these three infectious diseases because primary school children are an important reservoir of infection and at risk of morbidity. The aim of the present study was to assess the prevalence of infection for A. lumbricoides, T. trichiura and Cryptosporidium, and associated risk factors among elementary school children in this region, to provide a scientific basis for the formulation of government health policies and the promotion of school health education to reduce the prevalence of the three parasitic diseases in school-age children. 
Table 1. Compilation of Ascariasis, Trichuriasis and Cryptosporidiosis with relevance in Asia and China.

\begin{tabular}{|c|c|c|c|c|c|c|}
\hline Disease & Organism Types & Disease Agent & $\begin{array}{l}\text { Transmission Pathway } \\
\text { Related to Water or Soil }\end{array}$ & Risk Factors & Sanitation and Hygiene & Relevance \\
\hline Ascariasis & Secernentea & $\begin{array}{c}\text { Ascaris } \\
\text { lumbricoides }\end{array}$ & $\begin{array}{l}\text { Fertilized eggs in the moist } \\
\text { soil become infectious } \\
\text { about two weeks later. } \\
\text { Humans are infected } \\
\text { through contaminated } \\
\text { water and soil [16]. }\end{array}$ & \multirow{2}{*}{$\begin{array}{l}\text { Preschool children and } \\
\text { school age children; } \\
\text { Hypoimmunity; } \\
\text { Overcrowding; Poverty; } \\
\text { Using stools to make } \\
\text { fertilizer; Poor health } \\
\text { education [16]. }\end{array}$} & \multirow{2}{*}{$\begin{array}{l}\text { Poor living conditions, } \\
\text { (e.g., living with } \\
\text { livestock), improper } \\
\text { hygienic, and sanitary } \\
\text { practices, (e.g., eating raw } \\
\text { food or drinking raw, } \\
\text { water) will increase the } \\
\text { morbidity of STH }[17,18] .\end{array}$} & \multirow{2}{*}{$\begin{array}{l}\text { Worldwide, more than } 1.5 \text { billion } \\
\text { people ( } 24 \% \text { of the world's } \\
\text { population) are infected with STH, } \\
\text { mainly including Ascariasis } \\
\text { ( } 819.0 \text { million), huriasTricis } \\
\text { ( } 464.6 \text { million), and hookworm } \\
\text { infection ( } 438.9 \text { million). Nearly } \\
70 \% \text { of the global STH burden } \\
\text { occur in Asia [4]. Results of } \\
\text { China's second national survey for } \\
\text { STH showed that the prevalence of } \\
\text { A. lumbricoides and T. trichiura were } \\
12.7 \% \text { and } 4.6 \% \text {, respectively [19]. }\end{array}$} \\
\hline Trichuriasis & Adenophorea & Trichuris trichiura & $\begin{array}{l}\text { Immature eggs in soil } \\
\text { under favorable conditions } \\
\text { take about three weeks to } \\
\text { mature. Humans are } \\
\text { infected through } \\
\text { contaminated water and } \\
\text { soil [16]. }\end{array}$ & & & \\
\hline Cryptosporidiosis & Apicomplexan & $\begin{array}{l}\text { Cryptosporidium } \\
\text { spp. }\end{array}$ & $\begin{array}{l}\text { Cryptosporidium is } \\
\text { transmitted via } \\
\text { contaminated water or } \\
\text { food, swimming or bathing } \\
\text { in surface waters [6]. }\end{array}$ & $\begin{array}{l}\text { Animal contact; Children } \\
\text { from } 1 \text { to } 9 \text { years old; } \\
\text { Hypoimmunity Both } \\
\text { high ambient } \\
\text { temperature and high } \\
\text { rainfall. Malnutrition } \\
\text { Overcrowding [20]. }\end{array}$ & $\begin{array}{l}\text { Inadequate sanitation } \\
\text { and safe drinking water } \\
\text { coverage will increase the } \\
\text { transmission of } \\
\text { Cryptosporidium [20]. }\end{array}$ & $\begin{array}{l}\text { Cryptosporidiosis is an important } \\
\text { cause of diarrhea disease, more } \\
\text { common in developing countries } \\
(5 \% \text { to }>10 \%) \text { than in developed } \\
\text { countries }(<1-3 \%) \text { [6]. In China, } \\
\text { the proportion of diarrhea cases } \\
\text { caused by Cryptosporidium varies } \\
\text { between } 1.4 \% \text { to } 10.4 \% \text { and } \\
\text { prevalence among the general } \\
\text { population range from } 0.79 \% \text { to } \\
6.59 \% \text { [21]. }\end{array}$ \\
\hline
\end{tabular}




\section{Materials and Methods}

\subsection{Study Area}

The survey was conducted from 23 October to 3 November 2014 in Lw Primary School (Figure 1), Lw town, Puge County of the Liangshan Prefecture, southwestern China. Lw town features a complex topography of mountains and valleys with an average elevation of $1800 \mathrm{~m}$. The regional climate is subtropical monsoon, characterized by mild winters and warm and humid summers. The annual rainfall is $900 \mathrm{~mm}$ with the main rainy season from May to October and occasional rain throughout the rest of a year. The average temperature for the region is approximately $20{ }^{\circ} \mathrm{C}$. The local population is approximately 3000, most of whom are of the Yi ethnicity. The region is one of China's state-level poverty-stricken areas. Agriculture and animal husbandry are vital sources of household income. Unprotected contact with animals often causes local people to suffer from various zoonotic diseases.

At the time of the study, Liangshan Prefecture had poor sanitary conditions, especially at home, characterized by unsterilized drinking water and inadequate access to lavatories.

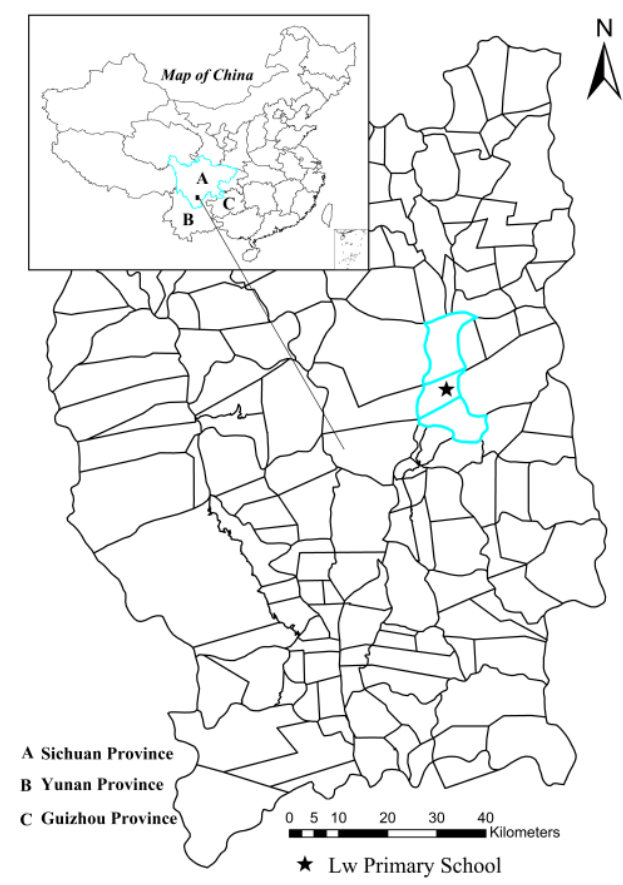

Figure 1. Map of the study area, showing Lw Primary School in Lw town, Puge County.

\subsection{Participant Recruitment and Field Study Procedures}

Schools are convenient sites for field surveys and school-age children are at high risk of helminths and protozoan infections. Lw Primary School of Lw town was selected with the help of the local health officials and all children from Grades 3, 4, 5 and 6 were selected for the survey. These four grades are selected because they are old enough have sufficient ability and good compliance to understand and complete the questionnaire. School teachers and the local health officials of the Center for Disease Control and Prevention (CDC) were invited to a sensitization meeting prior to our surveys. The study procedures and the structure of the questionnaire were explained in detail under the guidance of a unified protocol at the meeting. Health workers and teachers then informed all potential participants based on the protocol list and carefully explained the research objectives, procedures, and potential risks. A written informed consent form was obtained from the parents/guardians of the eligible students. A questionnaire and a clean plastic bag were distributed to the students by school teachers. Trained workers asked all participants carefully to ensure that each question was answered. Each participant was given a unique identification number. 


\subsection{Laboratory Procedures and Survey Measures}

Stool samples were collected once for each participant and sent to the laboratory of the local CDC as soon as possible. For the diagnosis of helminth infection, three Kato-Katz thick smear slides were prepared within $24 \mathrm{~h}$ post-collection. Another two thin smears were prepared using modified acid-fast staining to detect the oocysts of Cryptosporidium. All the slides were read by two independent examiners and a third examiner was called in if there was a disagreement. The survey included information on demographic characteristics, risk factors, and sanitation conditions, which were associated with those three infectious diseases. Demographic characteristics data of school children included gender, ethnicity, age, and the number of family members. Risk factors of infections were based on four major items, including washing hands, eating raw food or drinking raw water, the place to eat, and engaging in farm work. Sanitation associated with the student's home were assessed by two items, including water sources and types of lavatories.

\subsection{Ethical Considerations}

This study was approved by the Ethics Review Committee of the Ethical Institute of the School of Public Health, Fudan University (reference No. \#2010-08-0235). After the survey, all children with infection were offered free anti-helminthic treatment according to the national guidelines.

\section{Statistical Analysis}

Data were checked and entered into EpiData version 3.1 (EpiData Assoc., Odense, Denmark) and internal consistency checks were done. Statistical analyses were carried out with the SAS version 9.4 software (SAS Institute, Cary, NC, USA). Descriptive summary of the children's characteristics was computed as appropriate. Prevalence of A. lumbricoides and T. trichiura and Cryptosporidium infections with the $95 \%$ confidence interval were calculated. Fisher's exact test was used to compare the prevalence of the three parasitic diseases in different age groups. Univariate and multivariate analyses were performed to identify risk factors for the three parasitic diseases and any intestinal infection (any intestinal infection refers to the infection with one or more of those three infectious diseases). In the univariate analyses, Pearson's $\chi^{2}$ test was used to examine the associations of participants' characteristics with A. lumbricoides and T. trichiura and Cryptosporidium infections by computing crude odds ratios (ORs) with $95 \%$ confidence intervals (CIs). A subsequent multivariate logistic regression model was employed to identify risk factors and adjusted ORs with $95 \%$ CIs were calculated. Since a correlation existed between living on campus and eating at the school canteen, have a household lavatory and types of lavatories at home, only one of the two related variables was included in the multivariate model at a time. A two-sided $p$-value of 0.05 or less was regarded as significant.

\section{Results}

There were a total of 348 eligible participants in the study. After exclusion of participants who did not provide feces or complete the questionnaires, 321 (92.2\%) participants were included in the analysis. Demographic data showed similar distributions for those included and not included in the analysis (Table 2). 
Table 2. Distribution of demographic factors for participants included and not included in the analysis ${ }^{\text {a }}$.

\begin{tabular}{|c|c|c|c|}
\hline Characteristics & $\begin{array}{l}\text { Participants Included in } \\
\text { the Analysis }(n=321)\end{array}$ & $\begin{array}{l}\text { Participants not Included in the } \\
\text { Analysis }(n=27)\end{array}$ & $p$-Value \\
\hline Age $^{b}$ & $12.47 \pm 1.91$ & $13.11 \pm 1.85$ & 0.093 \\
\hline Male sex & $202(62.93 \%)$ & $12(44.44 \%)$ & 0.058 \\
\hline No. of family members $(\geq 6)$ & $254(79.13)$ & $19(70.37 \%)$ & 0.288 \\
\hline
\end{tabular}

Table 3 shows the prevalence of A. lumbricoides, T. trichiura, Cryptosporidium and coinfections by age group. The prevalence of infection was 10.0\% (95\% CI: $6.9-13.8 \%$ ) for A. lumbricoides, $25.2 \%$ (95\% CI: 20.6-30.4\%) for T. trichiura and 2.4\% (95\% CI: 1.1-4.9\%) for Cryptosporidium. The prevalence of co-infection was 3.7\% (95\% CI: 1.9-6.4\%) for A. lumbricoides / T. trichiura, $0.3 \%$ (95\% CI: 0-1.7\%) for A. lumbricoides /Cryptosporidium and 0.9\% (95\% CI: 0.2-2.7\%) for T. trichiura/Cryptosporidium. No triple infection was found in our study. The prevalence of A. lumbricoides infection differed among the age groups and was the lowest among children over 13 years of age $(p=0.04)$, while T. trichiura and Cryptosporidium infections and all the co-infections were not significantly different among the age groups (Table 3).

Table 3. Prevalence of A. lumbricoides, T. trichiura, Cryptosporidium and coinfections by age group a ${ }^{\text {. }}$

\begin{tabular}{cccccc}
\hline Characteristics & Total & $<\mathbf{1 2}$ Years & $\mathbf{1 2 - 1 3}$ Years & $>\mathbf{1 3}$ Years & $p$-Value \\
\hline A. lumbricoides infection & $10.0(6.9-13.8)$ & $12.4(6.3-21.0)$ & $13.0(7.7-20.0)$ & $4.0(1.1-8.8)$ & 0.039 \\
T. trichiura infection & $25.2(20.6-30.4)$ & $28.1(19.1-38.6)$ & $19.1(12.7-26.9)$ & $30.7(21.9-40.7)$ & 0.100 \\
Cryptosporidium infection & $2.4(1.1-4.9)$ & $2.2(0.3-7.9)$ & $3.8(1.2-8.7)$ & $1.0(0.0-5.4)$ & 0.394 \\
Coinfection & & & & \\
A. lumbricoides/T. trichiura & $3.7(1.9-6.4)$ & $5.6(1.8-12.6)$ & $4.6(1.7-9.7)$ & $1.0(0-5.4)$ & 0.164 \\
A. lumbricoides/Cryptosporidium & $0.3(0-1.7)$ & 0 & $0.8(0-4.2)$ & 0 & 1.000 \\
T. Trichiura/Cryptosporidium & $0.9(0.2-2.7)$ & 0 & $1.5(0.2-5.4)$ & $1.0(0-5.4)$ & 0.784 \\
\hline
\end{tabular}

${ }^{a}$ Values are infection rates with $95 \%$ confidence intervals and compared using the Fisher's exact test.

The characteristics of the participants are described in Table 4. The mean age of participants was 12.6 years (SD: 2.0). Among 321 children recruited, $62.93 \%$ were male; $98.75 \%$ were Yi people; $89.10 \%$ lived on campus and had their three meals of week days at the school canteen. At homes of the 321 participants recruited, $85.98 \%$ of the drinking water came from mountain springs, $60 \%$ of household lavatories were simple, and 30\% were triple compartment or biogas pools.

Table 4. Description of the participants by age group. Data are number (\%) of participants unless otherwise indicated.

\begin{tabular}{|c|c|c|c|c|}
\hline Characteristics & $<12$ Years $(n=89)$ & 12-13 Years $(n=131)$ & $>13$ Years $(n=101)$ & Total $(n=321)$ \\
\hline \multicolumn{5}{|l|}{ Sex } \\
\hline Female & $39(43.82)$ & $48(36.64)$ & $32(31.68)$ & 119 (37.07) \\
\hline Male & $50(56.18)$ & $83(63.36)$ & $69(68.32)$ & $202(62.93)$ \\
\hline \multicolumn{5}{|l|}{ No. of family members } \\
\hline$<6$ & $18(20.22)$ & $24(18.32)$ & $25(24.75)$ & $67(20.87)$ \\
\hline$\geq 6$ & $71(79.78)$ & 107 (81.68) & $76(75.25)$ & $254(79.13)$ \\
\hline \multicolumn{5}{|l|}{ Ethnicity } \\
\hline Han & $1(1.12)$ & $2(1.53)$ & $1(0.99)$ & $4(1.25)$ \\
\hline Yi & $88(98.88)$ & $129(98.47)$ & $100(99.01)$ & 317 (98.75) \\
\hline \multicolumn{5}{|c|}{ Having domestic animals } \\
\hline No & $1(1.12)$ & $0(0.00)$ & $5(4.95)$ & $6(1.87)$ \\
\hline Yes & $88(98.88)$ & $131(100.00)$ & $96(95.05)$ & $315(98.13)$ \\
\hline
\end{tabular}


Table 4. Cont

\begin{tabular}{|c|c|c|c|c|}
\hline Characteristics & $<12$ Years $(n=89)$ & $12-13$ Years $(n=131)$ & $>13$ Years $(n=101)$ & Total $(n=321)$ \\
\hline \multicolumn{5}{|c|}{ Drinking unboiled water at home } \\
\hline No or Occasionally & $5(5.62)$ & $22(16.79)$ & $23(22.77)$ & $50(15.58)$ \\
\hline Always & $84(94.38)$ & $109(83.21)$ & $78(77.23)$ & $271(84.42)$ \\
\hline \multicolumn{5}{|l|}{ Water sources at home } \\
\hline Springs & $70(78.65)$ & $117(89.31)$ & $88(87.13)$ & $275(85.67)$ \\
\hline Wells or rivers & $19(21.35)$ & $14(10.69)$ & $13(12.87)$ & $46(14.33)$ \\
\hline \multicolumn{5}{|c|}{ Washing hands before meals } \\
\hline No & $11(12.36)$ & $7(5.34)$ & $5(4.95)$ & $23(7.17)$ \\
\hline Occasionally & $62(69.66)$ & $90(68.70)$ & $81(80.20)$ & $233(72.59)$ \\
\hline Always & $16(17.98)$ & $34(25.95)$ & $15(14.85)$ & $65(20.25)$ \\
\hline \multicolumn{5}{|c|}{ Washing hands after defecation } \\
\hline No & $36(40.45)$ & $27(20.61)$ & $13(12.87)$ & $76(23.68)$ \\
\hline Occasionally & $43(48.31)$ & $64(48.85)$ & $62(61.39)$ & $169(56.65)$ \\
\hline Always & $10(11.24)$ & $40(30.53)$ & $26(25.74)$ & $76(23.68)$ \\
\hline \multicolumn{5}{|l|}{ Eating raw food at home } \\
\hline No & $14(15.73)$ & $18(13.74)$ & $11(10.89)$ & $43(13.40)$ \\
\hline Yes & $75(84.27)$ & $113(86.26)$ & $90(89.11)$ & $278(86.60)$ \\
\hline \multicolumn{5}{|l|}{ Engaging in farm work } \\
\hline No & $21(23.60)$ & $50(38.17)$ & $33(32.67)$ & $104(32.40)$ \\
\hline Yes & $68(76.40)$ & $81(61.83)$ & $68(67.33)$ & $217(67.60)$ \\
\hline \multicolumn{5}{|c|}{ Using human or animal feces as fertilizer } \\
\hline No & $5(5.62)$ & $5(3.82)$ & $7(6.93)$ & $17(5.30)$ \\
\hline Yes & $84(94.38)$ & $126(96.18)$ & $94(93.07)$ & $304(94.70)$ \\
\hline \multicolumn{5}{|c|}{ Having a household lavatory at home } \\
\hline No & $40(44.94)$ & $39(29.77)$ & $33(32.67)$ & $112(34.89)$ \\
\hline Yes & $49(55.06)$ & $92(70.23)$ & $68(67.33)$ & $209(65.11)$ \\
\hline \multicolumn{5}{|l|}{ Type of lavatory at home } \\
\hline No & $40(44.94)$ & $39(29.77)$ & $33(32.67)$ & $112(34.89)$ \\
\hline Simple & $34(38.20)$ & $61(46.56)$ & $42(41.58)$ & $137(42.68)$ \\
\hline $\begin{array}{c}\text { Triple compartment or } \\
\text { biogas pools }\end{array}$ & $15(16.85)$ & $31(23.66)$ & $26(25.74)$ & $72(22.43)$ \\
\hline \multicolumn{5}{|l|}{ Eating at the school canteen } \\
\hline No & $2(2.25)$ & $5(3.82)$ & $4(3.96)$ & $11(3.43)$ \\
\hline Only lunch & $10(11.24)$ & $7(5.34)$ & $7(6.93)$ & $24(7.48)$ \\
\hline Three meals & $77(86.52)$ & $119(90.84)$ & $90(89.11)$ & $286(89.10)$ \\
\hline \multicolumn{5}{|l|}{ Living on campus } \\
\hline No & $12(13.48)$ & $12(9.16)$ & $11(10.89)$ & $35(10.90)$ \\
\hline Yes & $77(86.52)$ & $119(90.84)$ & $90(89.11)$ & $286(89.10)$ \\
\hline
\end{tabular}

Tables 5 and 6 show the results of risk factors associated with A. lumbricoides, T. trichiura and Cryptosporidium infections. Children from households using well or river water were at a greater risk of A. lumbricoides infection ( $\mathrm{aOR}=2.67,95 \% \mathrm{CI}: 1.15-6.20)$. Having a household lavatory was negatively associated with T. trichiura infection ( $\mathrm{aOR}=0.50,95 \% \mathrm{CI}$ : $0.30-0.84$ ). Children having their three meals at the school canteen were at a lower risk of Cryptosporidium infection (aOR $=0.06,95 \% \mathrm{CI}: 0.01-0.40)$. Similarly, living on campus was also a protective factor for Cryptosporidium infection ( $\mathrm{COR}=0.11,95 \%$ CI: $0.03-0.46)$. Using spring water as water source was a protective factor for any intestinal infection $(\mathrm{aOR}=0.52,95 \%$ CI: $0.28-0.99)$. 
Table 5. Univariable and multivariable analysis for the associations between potential risk factors and A. lumbricoides and T. trichiura infections.

\begin{tabular}{|c|c|c|c|c|c|c|c|c|c|c|c|}
\hline \multirow[b]{2}{*}{ Characteristics } & \multirow[b]{2}{*}{$\begin{array}{c}\text { No. of } \\
\text { Tested (\%) }\end{array}$} & \multicolumn{5}{|c|}{ A. lumbricoides } & \multicolumn{5}{|c|}{ T. trichiura } \\
\hline & & $\begin{array}{c}\text { No. of } \\
\text { Infections (\%) }\end{array}$ & cOR $(95 \% \mathrm{CI})$ & $p$-Value & $\mathrm{aOR}(95 \% \mathrm{CI})$ & $p$-Value & $\begin{array}{c}\text { No. of } \\
\text { Infections (\%) }\end{array}$ & $\operatorname{cOR}(95 \% \mathrm{CI})$ & $p$-Value & aOR $(95 \% \mathrm{CI})$ & $p$-Value \\
\hline \multicolumn{12}{|l|}{ Sex } \\
\hline Female & $119(37.07)$ & $16(12.61)$ & 1.00 & & & & $31(26.05)$ & 1.00 & & & \\
\hline Male & $202(62.93)$ & $17(8.42)$ & $0.64(0.31-1.33)$ & 0.236 & & & $50(24.75)$ & $0.93(0.56-1.57)$ & 0.794 & & \\
\hline \multicolumn{12}{|l|}{ Age group } \\
\hline$<12$ & $96(29.91)$ & $11(12.36)$ & 1.00 & & & & $25(28.09)$ & 1.00 & & & \\
\hline $12-13$ & $140(43.61)$ & $17(12.98)$ & $1.06(0.47-2.38)$ & 0.902 & & & $25(19.08)$ & $0.60(0.32-1.14)$ & 0.124 & & \\
\hline$>13$ & $117(36.45)$ & $4(3.96)$ & $0.29(0.09-0.95)$ & 0.037 & & & $31(30.69)$ & $1.13(0.61-2.12)$ & 0.699 & & \\
\hline \multicolumn{12}{|l|}{ No. of family members } \\
\hline$<6$ & $67(20.87)$ & $1(5.97)$ & 1.00 & & & & $14(20.90)$ & 1.00 & & & \\
\hline$\geq 6$ & $254(79.13)$ & $28(11.02)$ & $1.95(0.66-5.77)$ & 0.224 & & & $67(26.38)$ & $1.36(0.71-2.60)$ & 0.365 & & \\
\hline \multicolumn{12}{|c|}{ Drinking unboiled water at home } \\
\hline No or Occasionally & $50(15.58)$ & $4(8.00)$ & 1.00 & & & & $9(18.00)$ & 1.00 & & & \\
\hline Always & $271(84.42)$ & $28(10.33)$ & $1.32(0.44-3.96)$ & 0.649 & & & $72(26.57)$ & $1.65(0.76-3.56)$ & 0.202 & & \\
\hline \multicolumn{12}{|l|}{ Water sources at home } \\
\hline Springs & $276(85.98)$ & $23(8.33)$ & 1.00 & & 1.00 & & $66(23.91)$ & 1.00 & & & \\
\hline Wells or rivers & 45 (14.02) & $9(20.00)$ & $2.67(1.15-6.20)$ & 0.033 & $2.67(1.15-6.20)$ & 0.033 & 15 (33.33) & $1.59(0.81-3.14)$ & 0.224 & & \\
\hline \multicolumn{12}{|c|}{ Washing hands before meals } \\
\hline No & $23(7.17)$ & $4(17.39)$ & 1.00 & & & & $5(21.74)$ & 1.00 & & & \\
\hline Occasionally & $233(72.59)$ & $19(8.15)$ & $0.42(0.13-1.37)$ & 0.179 & & & $60(25.75)$ & $1.25(0.44-3.51)$ & 0.703 & & \\
\hline Always & $65(20.25)$ & $9(13.85)$ & $0.76(0.21-2.77)$ & 0.677 & & & $16(24.62)$ & $1.18(0.38-3.68)$ & 0.806 & & \\
\hline \multicolumn{12}{|c|}{ Washing hands after defecation } \\
\hline No & $76(23.68)$ & $10(13.16)$ & 1.00 & & & & $17(22.37)$ & 1.00 & & & \\
\hline Occasionally & $169(56.65)$ & $14(8.28)$ & $0.60(0.25-1.41)$ & 0.250 & & & $50(29.59)$ & $0.60(0.25-1.41)$ & 0.245 & & \\
\hline Always & $76(23.68)$ & $8(10.53)$ & $0.78(0.29-2.09)$ & 0.627 & & & 14 (18.42) & $0.78(0.29-2.10)$ & 0.554 & & \\
\hline \multicolumn{12}{|l|}{ Eating raw food at home } \\
\hline No & $43(13.40)$ & $6(13.95)$ & 1.00 & & & & $10(23.26)$ & 1.00 & & & \\
\hline Yes & $278(86.60)$ & $26(9.35)$ & $0.64(0.25-1.65)$ & 0.360 & & & $71(25.54)$ & $1.13(0.53-2.41)$ & 0.768 & & \\
\hline \multicolumn{12}{|c|}{ Engaging in farm work at home } \\
\hline No & $104(32.40)$ & $7(5.77)$ & 1.00 & & & & $28(26.92)$ & 1.00 & & & \\
\hline Yes & $217(67.60)$ & $26(11.98)$ & $2.22(0.89-5.58)$ & 0.080 & & & $53(24.42)$ & $0.88(0.52-1.49)$ & 0.629 & & \\
\hline \multicolumn{12}{|c|}{ Using human or animal feces as fertilizer } \\
\hline No & $17(5.30)$ & $1(5.88)$ & 1.00 & & & & $2(11.76)$ & 1.00 & & & \\
\hline Yes & $304(94.70)$ & $31(10.20)$ & $1.82(0.23-14.17)$ & 0.638 & & & $79(25.99)$ & $2.63(0.59-11.77)$ & 0.195 & & \\
\hline \multicolumn{12}{|l|}{ Having a lavatory at home } \\
\hline No & $112(34.89)$ & $11(9.82)$ & 1.00 & & & & 38 (33.93) & 1.00 & & 1.00 & \\
\hline Yes & 209 (65.11) & $21(10.05)$ & $1.03(0.48-2.21)$ & 0.962 & & & $43(20.57)$ & $0.50(0.30-0.84)$ & 0.010 & $0.50(0.30-0.84)$ & 0.010 \\
\hline
\end{tabular}


Table 5. Cont.

\begin{tabular}{|c|c|c|c|c|c|c|c|c|c|c|c|}
\hline \multirow[b]{2}{*}{ Characteristics } & \multirow{2}{*}{$\begin{array}{c}\text { No. of } \\
\text { Tested (\%) }\end{array}$} & \multicolumn{5}{|c|}{ A. lumbricoides } & \multicolumn{5}{|c|}{ T. trichiura } \\
\hline & & $\begin{array}{c}\text { No. of } \\
\text { Infections (\%) }\end{array}$ & $\operatorname{cOR}(95 \% \mathrm{CI})$ & $p$-Value & aOR $(95 \% \mathrm{CI})$ & $p$-Value & $\begin{array}{c}\text { No. of } \\
\text { Infections (\%) }\end{array}$ & cOR $(95 \% \mathrm{CI})$ & $p$-Value & aOR $(95 \%$ CI) & $p$-Value \\
\hline \multicolumn{12}{|l|}{ Types of lavatories at home } \\
\hline No & $112(34.89)$ & $11(9.82)$ & 1.00 & & & & $38(33.93)$ & 1.00 & & & \\
\hline Simple & $137(42.68)$ & $13(9.49)$ & $0.96(0.41-2.24)$ & 0.927 & & & $28(20.44)$ & $0.50(0.28-0.89)$ & 0.018 & & \\
\hline $\begin{array}{l}\text { Triple compartment or biogas } \\
\text { pools }\end{array}$ & $72(22.43)$ & $8(11.11)$ & $1.15(0.44-3.01)$ & 0.777 & & & $15(20.83)$ & $0.51(0.26-1.02)$ & 0.057 & & \\
\hline \multicolumn{12}{|l|}{ Living on campus } \\
\hline No & $35(10.90)$ & $3(8.57)$ & 1.00 & & & & $7(20.00)$ & 1.00 & & & \\
\hline Yes & $286(89.10)$ & $29(10.14)$ & $1.20(0.35-4.18)$ & 0.821 & & & $74(25.87)$ & $1.40(0.59-3.33)$ & 0.467 & & \\
\hline \multicolumn{12}{|l|}{ Eating at the school canteen } \\
\hline No & $11(3.43)$ & $1(9.09)$ & 1.00 & & & & $1(9.09)$ & 1.00 & & & \\
\hline Only lunch & $24(7.48)$ & $2(8.33)$ & $0.91(0.07-11.23)$ & 0.918 & & & $6(25.00)$ & $3.33(0.35-31.70)$ & 0.323 & & \\
\hline Three meals & $286(89.10)$ & $29(9.97)$ & $1.13(0.14-9.13)$ & 0.996 & & & $74(25.87)$ & $3.49(0.44-27.69)$ & 0.225 & & \\
\hline \multicolumn{12}{|l|}{ A. lumbricoides } \\
\hline No & $289(90.03)$ & - & - & & & & $69(23.88)$ & 1.00 & & & \\
\hline Yes & $32(9.97)$ & - & - & & & & $12(37.50)$ & $1.91(0.89-4.11)$ & 0.142 & & \\
\hline \multicolumn{12}{|l|}{ T. trichiura } \\
\hline No & $240(74.77)$ & $20(8.33)$ & 1.00 & & & & - & - & & & \\
\hline Yes & $81(25.23)$ & $12(14.81)$ & $1.91(0.89-4.11)$ & 0.107 & & & - & - & & & \\
\hline \multicolumn{12}{|l|}{ Cryptosporidium } \\
\hline No & $313(97.51)$ & $31(9.90)$ & 1.00 & & & & $78(24.92)$ & 1.00 & & & \\
\hline Yes & $8(2.49)$ & $1(12.50)$ & $1.30(0.15-10.91)$ & 0.757 & & & $3(37.50)$ & $1.81(0.42-7.74)$ & 0.441 & & \\
\hline
\end{tabular}


Table 6. Univariable and multivariable analysis for the association between potential risk factors and Cryptosporidium and any intestinal infection.

\begin{tabular}{|c|c|c|c|c|c|c|c|c|c|c|c|}
\hline \multirow[b]{2}{*}{ Characteristics } & \multirow[b]{2}{*}{$\begin{array}{c}\text { No. of } \\
\text { Tested (\%) }\end{array}$} & \multicolumn{5}{|c|}{ Cryptosporidium } & \multicolumn{5}{|c|}{ Any intestinal Infection } \\
\hline & & $\begin{array}{c}\text { No. of } \\
\text { Infections (\%) }\end{array}$ & $\operatorname{cOR}(95 \% \mathrm{CI})$ & $p$-Value & aOR $(95 \% \mathrm{CI})$ & $p$-Value & $\begin{array}{c}\text { No. of } \\
\text { Infections (\%) }\end{array}$ & cOR $(95 \%$ CI $)$ & $p$-Value & aOR $(95 \% \mathrm{CI})$ & $p$-Value \\
\hline \multicolumn{12}{|l|}{ Sex } \\
\hline Female & $119(37.07)$ & $4(3.36)$ & 1.00 & & & & $43(36.13)$ & 1.00 & & & \\
\hline Male & $202(62.93)$ & $4(1.98)$ & $0.58(0.14-2.37)$ & 0.448 & & & $62(30.69)$ & $0.78(0.49-1.26)$ & 0.316 & & \\
\hline \multicolumn{12}{|l|}{ Age group } \\
\hline$<12$ & $96(29.91)$ & $2(2.25)$ & 1.00 & & & & $33(37.08)$ & 1.00 & & & \\
\hline $12-13$ & $140(43.61)$ & $5(3.82)$ & $1.73(0.33-9.10)$ & 0.520 & & & $38(29.01)$ & $0.69(0.47-1.56)$ & 0.210 & & \\
\hline$>13$ & $117(36.45)$ & $1(0.99)$ & $0.44(0.04-4.88)$ & 0.500 & & & $34(33.66)$ & $0.86(0.39-1.23)$ & 0.623 & & \\
\hline \multicolumn{12}{|l|}{ No. of family members } \\
\hline$<6$ & $67(20.87)$ & $1(1.49)$ & 1.00 & & & & $17(25.37)$ & 1.00 & & & \\
\hline$\geq 6$ & $254(79.13)$ & $7(2.76)$ & $1.87(0.23-15.47)$ & 0.561 & & & $88(34.65)$ & $1.56(0.85-2.87)$ & 0.152 & & \\
\hline \multicolumn{12}{|l|}{ Water sources at home } \\
\hline Wells or rivers & 45 (14.02) & $1(2.22)$ & 1.00 & & & & $20(44.44)$ & 1.00 & & & \\
\hline Springs & $276(85.98)$ & $7(2.54)$ & $1.18(0.14-9.78)$ & 0.881 & & & $85(30.80)$ & $0.52(0.28-0.99)$ & 0.046 & $0.52(0.28-0.99)$ & 0.046 \\
\hline \multicolumn{12}{|c|}{ Drinking unboiled water at home } \\
\hline No or Occasionally & $50(15.58)$ & $2(4.00)$ & 1.00 & & & & $13(26.00)$ & 1.00 & & & \\
\hline Always & $271(84.42)$ & $6(2.14)$ & $1.84(0.36-9.39)$ & 0.463 & & & $92(33.95)$ & $1.46(0.74-2.89)$ & 0.273 & & \\
\hline \multicolumn{12}{|l|}{ Wash hands before meals } \\
\hline No & $23(7.17)$ & $1(4.35)$ & 1.00 & & & & $9(39.13)$ & 1.00 & & & \\
\hline Occasionally & $233(72.59)$ & $6(2.58)$ & $0.58(0.07-5.05)$ & 0.623 & & & $75(32.19)$ & $0.74(0.31-1.78)$ & 0.500 & & \\
\hline Always & $65(20.25)$ & $1(1.54)$ & $0.34(0.02-5.73)$ & 0.457 & & & $21(32.31)$ & $0.74(0.28-1.99)$ & 0.554 & & \\
\hline \multicolumn{12}{|l|}{ Wash hands after defecation } \\
\hline No & $76(23.68)$ & $2(2.63)$ & 1.00 & & & & $26(34.21)$ & 1.00 & & & \\
\hline Occasionally & $169(56.65)$ & $2(1.18)$ & $0.44(0.06-3.21)$ & 0.420 & & & 57 (33.73) & $0.98(0.55-1.73)$ & 0.941 & & \\
\hline Always & $76(23.68)$ & $4(5.26)$ & $2.06(0.37-11.57)$ & 0.414 & & & $22(28.95)$ & $0.79(0.45-1.40)$ & 0.486 & & \\
\hline \multicolumn{12}{|l|}{ Eating raw food at home } \\
\hline No & $43(13.40)$ & $2(4.65)$ & 1.00 & & & & $16(37.21)$ & 1.00 & & & \\
\hline Yes & $278(86.60)$ & $6(2.16)$ & $0.45(0.09-2.32)$ & 0.341 & & & $89(32.01)$ & $0.76(0.41-1.55)$ & 0.500 & & \\
\hline \multicolumn{12}{|l|}{ Engaging in farm work } \\
\hline No & $104(32.40)$ & $2(1.92)$ & 1.00 & & & & $32(30.77)$ & 1.00 & & & \\
\hline Yes & $217(67.60)$ & $6(2.76)$ & $1.45(0.29-7.31)$ & 0.652 & & & $73(33.64)$ & $1.14(0.69-1.89)$ & 0.877 & & \\
\hline \multicolumn{12}{|c|}{ Using human or animal feces as fertilizer } \\
\hline No & $17(5.30)$ & $0(0.00)$ & 1.00 & & & & $3(17.65)$ & 1.00 & & & \\
\hline Yes & $304(94.70)$ & $8(2.63)$ & $1.00(0.06-18.10)$ & 0.999 & & & $102(33.55)$ & $2.36(0.66-8.39)$ & 0.186 & & \\
\hline \multicolumn{12}{|l|}{ Having a lavatory at home } \\
\hline No & $112(34.89)$ & $3(2.68)$ & 1.00 & & & & $46(41.07)$ & 1.00 & & & \\
\hline Yes & $209(65.11)$ & $5(2.39)$ & $0.89(0.21-3.80)$ & 0.875 & & & $59(28.23)$ & $0.56(0.35-0.91)$ & 0.020 & & \\
\hline
\end{tabular}


Table 6. Cont

\begin{tabular}{|c|c|c|c|c|c|c|c|c|c|c|c|}
\hline \multirow[b]{2}{*}{ Characteristics } & \multirow{2}{*}{$\begin{array}{c}\text { No. of } \\
\text { Tested (\%) }\end{array}$} & \multicolumn{5}{|c|}{ Cryptosporidium } & \multicolumn{5}{|c|}{ Any intestinal Infection } \\
\hline & & $\begin{array}{c}\text { No. of } \\
\text { Infections (\%) }\end{array}$ & cOR $(95 \% \mathrm{CI})$ & $p$-Value & aOR $(95 \%$ CI $)$ & $p$-Value & $\begin{array}{c}\text { No. of } \\
\text { Infections (\%) }\end{array}$ & cOR $(95 \% \mathrm{CI})$ & $p$-Value & aOR $(95 \%$ CI $)$ & $p$-Value \\
\hline \multicolumn{12}{|l|}{ Types of lavatories at home } \\
\hline No & $112(34.89)$ & $3(2.68)$ & 1.00 & & & & $46(41.07)$ & 1.00 & & & \\
\hline Simple & 137 (42.68) & $3(2.19)$ & $0.81(0.16-4.11)$ & 0.803 & & & $38(27.74)$ & $0.55(0.32-0.94)$ & 0.028 & & \\
\hline Triple compartment or biogas pools & $72(22.43)$ & $2(2.78)$ & $1.04(0.17-6.37)$ & 0.968 & & & 21 (29.17) & $0.59(0.31-1.11)$ & 0.103 & & \\
\hline \multicolumn{12}{|l|}{ Living on campus } \\
\hline No & $35(10.90)$ & $4(11.43)$ & 1.00 & & & & $12(34.29)$ & 1.00 & & & \\
\hline Yes & $286(89.1)$ & $4(1.40)$ & $0.11(0.03-0.46)$ & 0.003 & & & $93(32.52)$ & $0.92(0.44-1.94)$ & 0.833 & & \\
\hline \multicolumn{12}{|l|}{ Eating at the school canteen } \\
\hline No & $11(3.43)$ & $2(18.18)$ & 1.00 & & 1.00 & & $3(27.27)$ & 1.00 & & & \\
\hline Only lunch & $24(7.48)$ & $2(8.33)$ & $0.41(0.05-3.37)$ & 0.409 & $0.41(0.05-3.37)$ & 0.409 & $9(37.50)$ & $1.60(0.34-7.64)$ & 0.556 & & \\
\hline Three meals & $286(89.10)$ & $4(1.25)$ & $0.06(0.01-0.40)$ & 0.003 & $0.06(0.01-0.40)$ & 0.003 & $93(32.52)$ & $1.28(0.33-4.96)$ & 0.716 & & \\
\hline \multicolumn{12}{|l|}{ A. lumbricoides } \\
\hline No & $289(90.03)$ & $7(2.42)$ & 1.00 & & & & - & - & & & \\
\hline Yes & $32(9.97)$ & $1(3.13)$ & $1.30(0.16-10.91)$ & 0.809 & & & - & - & & & \\
\hline \multicolumn{12}{|l|}{ T. trichiura } \\
\hline No & $240(74.77)$ & $5(2.08)$ & 1.00 & & & & - & - & & & \\
\hline Yes & 81 (25.23) & $3(3.7)$ & $1.81(0.42-7.74)$ & 0.425 & & & - & - & & & \\
\hline
\end{tabular}




\section{Discussion}

This study reported the prevalence of A. lumbricoides, T. trichiura and Cryptosporidium infections in the elementary school children. The prevalence of Cryptosporidium infections in our study $(2.40 \%)$ was comparable to the provincial estimate (2.96\%) in Sichuan Province, China [15]. The prevalence of A. lumbricoides infection $(10.0 \%)$, was similar to the national prevalence $(12.7 \%)$ of the second national important parasitic disease survey from 2001 to 2004, but was lower than those of Guizhou Province $(42.0 \%)$ and Sichuan Province (27.7\%) [13]. Our study area is located in the southwestern of Sichuan Province, close to Yunnan and Guizhou Province (Figure 1). The reduced prevalence of A. lumbricoides infections may be related to the government's deworming treatment of $A$. lumbricoides in high-risk populations. A survey of school-age children in 2010 also showed a reduction in the prevalence of A. lumbricoides infection in rural southwestern China [18]. For T. trichiura infection, the prevalence in our study (25.2\%) was higher than both second national survey (4.6\% in China Mainland) and the survey of school-age children in southwestern China in 2010 (14.3\% in Guizhou Province, and 2.2\% in Sichuan Province) $[13,18]$. According to the WHO report, the prevalence of STH infection in China was estimated to be less than $20 \%$ [22]. The prevalence of T. trichiura infections in the study area remained high. The main reason may be the unsatisfactory efficacy of currently recommended drugs against T. trichiura infection, especially when they are not regularly taken [23]. Poor hygiene, especially at home, may be another reason for the high prevalence of $T$. trichiura infection. Most children in the school drank unboiled water $(84.42 \%)$ and ate raw food without washing $(86.60 \%)$. Water, food, soil and hands are easily contaminated by worm eggs in the case of inadequate sanitation facilities and feces management, unsafe water and poor hygiene practices [24]. Therefore, improving access to adequate water, sanitation and hygiene (WASH) at the household level and in school is essential to prevent infections of T. trichiura [25]. In addition, the climatic factors in Liangshan Prefecture are within the plausible limits of STH transmission [5]. As expected, the prevalence of A. lumbricoides infection varied by age. However, the age patterns of T. trichiura and Cryptosporidium infections showed little variation, which probably reflected a combination of behavioral and socioeconomic factors [26]. Indeed, older students had more opportunities to receive health education and knowledge about infection prevention. Interestingly, although most hygiene habits tended to improve with age in our study, older students were prone to eating raw food. This finding emphasized the importance of continuing and strengthening health education programs targeting school children to reduce the intensity of parasites and the attendant morbidity [12].

A recent meta-analysis of the effects of water, sanitation, and hygiene (WASH) on STH infections reported that access to piped water and proper water treatment was associated with lower risks of A. lumbricoides and T. trichiura infections [27]. Our study found that using river or well water was associated with an increased risk of $A$. lumbricoides infection. In the study area, the main sources of drinking water in the study area were mountain springs (far from human living areas, piped after extensive precipitation and filtration), followed by wells or rivers (both close to living areas and susceptible to fecal pollution). Moreover, some students' homes (14.33\%) used river or well water as the source of drinking water and $84.42 \%$ of school children were in the habit of drinking unboiled water. The drinking water is rarely disinfected and is often contaminated by untreated infectious human excreta, which puts the local residents at great risk of intestinal parasitic infections. Safer drinking water is an urgent need in the rural regions of Liangshan Prefecture. Having a household lavatory was negatively associated with $T$. trichiura infection, which was similar to previous findings [19,27-29]. In order to reduce intestinal infectious diseases, the local government promoted and constructed triple compartment toilets or biogas digesters that can fully ferment feces to kill the eggs and ensure that human waste is safely separated from human contact in some rural areas [30,31]. But many families only have simple lavatories that pile up human stools and urine as a direct fertilizer. A cluster-randomized trial undertaken in Kenya demonstrated that school hygiene and sanitation reduced the re-infections of STH after school-based deworming [2]. A recent meta-analysis has shown that adequate sanitation is associated with the reduction of STH transmission risk (between $40 \%$ 
and $50 \%$ reduced infection rates) [28]. There is no doubt that improvements to water, sanitation, and hygiene (WASH) lead to a significant reduction in STH infection. Schools are available platforms, not only for health education but for sanitation improvements. School-age children are more likely to receive health education and tend to integrate new health behaviors into their daily lives [32].

Soil-transmitted helminthiasis, a group of NTDs, is thought to be associated with malnutrition and iron deficiency anemia, and has a negative impact on children's physical and cognitive development [33]. Hence, reducing risk factors and regular deworming become important strategies for controlling STH [34]. Effective control of STH can have a positive outcome on students' education, as chronic helminth infection impedes cognitive development and leads to growth delays, thus delaying enrollment and grade progression [35].

Cryptosporidium is typically transmitted by fecal-oral routes, through ingestion of human or domestic animal fecal-contaminated food or untreated water. In our study, students having meals at the school canteen or living on campus had lower odds of obtaining Cryptosporidium infection. Students who live on campus $(89.10 \%)$ ate three meals at the school canteen. The elementary school we selected was basically a boarding school. The majority of the students stayed in the school dormitories for 5 days per week and returned home during holidays and weekends. One possible reason for the association of Cryptosporidium infection with eating in the school cafeteria was less chance of contact with domestic animals. Raising domestic animals is known as a risk factor for Cryptosporidium infection [36,37]. Another possible reason was that schools had better sanitation facilities and safer water, protecting those students who did not have access to these facilities at home $[14,32,38]$. The local government has been concerned about the safety of school drinking water. At Lw Primary School, improved water and sanitation facilities, including basic tap water, were available to the students. Tap water was used for handwashing without soap. A recent review showed that diarrhea and gastrointestinal diseases decreased as schools had access to adequate sanitation [39]. Improper waste disposal and unsafe water, especially at home, will increase the chance of transmission of fecal-oral diseases $[14,40]$.

We recognize a number of limitations in our study. The diagnoses of three intestinal parasitic infections were based on a single stool sample and underestimation of the infection rates might be possible. There was also a lack of data on helminth infection intensities and Cryptosporidium genotypes. Further research is needed to determine the infection intensities of A. lumbricoides and T. trichiura and also the routes of transmission of Cryptosporidium among school children in this region. To truly understand the motivators for behavior and exposure to infection, further studies might be needed to systematically assess risk factors of intestinal parasites by using a combination of quantitative and qualitative methods including water quality monitoring and WASH spot checks [40]. The sample size is not enough for more accurate statistical analysis. In view of these limitations, the findings should be interpreted discreetly.

\section{Conclusions}

In summary, our study revealed a high burden of A. lumbricoides and T. trichiura infections among elementary school children in southwestern China. School-level interventions involving deworming, health education, improvement of environmental sanitation and hygiene and access to safe water might be required. Schools are a good platform for health education, not only for students but also for their families. New methods of health education (e.g., comic and animation) might be explored. There is no doubt that improving health awareness among students can reduce their risky behaviors related to parasitic infections. Similarly, we should actively promote the reconstruction of harmless lavatory (e.g., triple compartment or biogas) in rural areas and improve the rural sanitary conditions, to reduce the transmission of intestinal infectious diseases. 
Author Contributions: Conceived and designed the investigation: Q.W.J. and Y.B.Z. Analyzed the data: Y.Y. (Ya Yang), D.J.Y. and Y.B.Z. Investigation and Data collection: Y.Y. (Ya Yang), Y.B.Z., D.J.Y., Y.Y. (Yu Yang), Y.J.W. and S.R.D. Wrote the paper: Y.Y. (Ya Yang), D.J.Y., Y.C. and Y.B.Z. The datasets supporting the conclusions of this article are included in the article.

Funding: This research did not receive any specific grant from funding agencies in the public, commercial, or not-for-profit sectors.

Acknowledgments: We thank all the school teachers, the local health officials for their assistance.

Conflicts of Interest: The authors declare no conflict of interest.

\section{References}

1. Bethony, J.; Brooker, S.; Albonico, M.; Geiger, S.M.; Loukas, A.; Diemert, D.; Hotez, P.J. Soil-transmitted helminth infections: Ascariasis, trichuriasis, and hookworm. Lancet 2006, 367, 1521-1532. [CrossRef]

2. Freeman, M.C.; Clasen, T.; Brooker, S.J.; Akoko, D.O.; Rheingans, R. The Impact of a School-Based Hygiene, Water Quality and Sanitation Intervention on Soil-Transmitted Helminth Reinfection: A Cluster-Randomized Trial. Am. J. Trop. Med. Hyg. 2013, 89, 875-883. [CrossRef] [PubMed]

3. WHO. Soil-Transmitted Helminth Infections. Available online: http://www.who.int/en/news-room/factsheets / detail/soil-transmitted-helminth-infections (accessed on 28 July 2018).

4. Pullan, R.L.; Smith, J.L.; Jasrasaria, R.; Brooker, S.J. Global numbers of infection and disease burden of soil transmitted helminth infections in 2010. Parasit. Vectors 2014, 7, 37. [CrossRef] [PubMed]

5. Pullan, R.L.; Brooker, S.J. The global limits and population at risk of soil-transmitted helminth infections in 2010. Parasit. Vectors 2012, 5, 81. [CrossRef] [PubMed]

6. Mahmoudi, M.R.; Ongerth, J.E.; Karanis, P. Cryptosporidium and cryptosporidiosis: The Asian perspective. Int. J. Hyg. Environ. Health 2017, 220, 1098-1109. [CrossRef] [PubMed]

7. Kotloff, K.L.; Nataro, J.P.; Blackwelder, W.C.; Nasrin, D.; Farag, T.H.; Panchalingam, S.; Wu, Y.; Sow, S.O.; Sur, D.; Breiman, R.F.; et al. Burden and aetiology of diarrhoeal disease in infants and young children in developing countries (the Global Enteric Multicenter Study, GEMS): A prospective, case-control study. Lancet 2013, 382, 209-222. [CrossRef]

8. Checkley, W.; White, A.J.; Jaganath, D.; Arrowood, M.J.; Chalmers, R.M.; Chen, X.M.; Fayer, R.; Griffiths, J.K.; Guerrant, R.L.; Hedstrom, L.; et al. A review of the global burden, novel diagnostics, therapeutics, and vaccine targets for cryptosporidium. Lancet Infect. Dis. 2015, 15, 85-94. [CrossRef]

9. Anthonj, C.; Rechenburg, A.; Hoser, C.; Kistemann, T. Contracting infectious diseases in Sub-Saharan African wetlands: A question of use? A review. Int. J. Hyg. Environ. Health 2017, 220, 1110-1123. [CrossRef] [PubMed]

10. Xu, J.; Xu, J.F.; Li, S.Z.; Zhang, L.J.; Wang, Q.; Zhu, H.H.; Zhou, X.N. Integrated control programmes for schistosomiasis and other helminth infections in P.R. China. Acta Trop. 2015, 141, 332-341. [CrossRef] [PubMed]

11. Shang, Y.; Tang, L.H.; Zhou, S.S.; Chen, Y.D.; Yang, Y.C.; Lin, S.X. Stunting and soil-transmitted-helminth infections among school-age pupils in rural areas of southern China. Parasit. Vectors 2010, 3, 97. [CrossRef] [PubMed]

12. Wang, X.; Zhang, L.; Luo, R.; Wang, G.; Chen, Y.; Medina, A.; Eggleston, K.; Rozelle, S.; Smith, D.S. Soil-transmitted helminth infections and correlated risk factors in preschool and school-aged children in rural Southwest China. PLoS ONE 2012, 7, e45939. [CrossRef] [PubMed]

13. Xiao, P.L.; Zhou, Y.B.; Chen, Y.; Yang, Y.; Shi, Y.; Gao, J.C.; Yihuo, W.L.; Song, X.X.; Jiang, Q.W. Prevalence and risk factors of Ascaris lumbricoides (Linnaeus, 1758), Trichuris trichiura (Linnaeus, 1771) and HBV infections in Southwestern China: A community-based cross sectional study. Parasit. Vectors 2015, 8, 661. [CrossRef] [PubMed]

14. Yap, P.; Du, Z.W.; Chen, R.; Zhang, L.P.; Wu, F.W.; Wang, J.; Wang, X.Z.; Zhou, H.; Zhou, X.N.; Utzinger, J.; et al. Soil-transmitted helminth infections and physical fitness in school-aged Bulang children in southwest China: Results from a cross-sectional survey. Parasit. Vectors 2012, 5, 50. [CrossRef] [PubMed]

15. Silver, Z.A.; Kaliappan, S.P.; Samuel, P.; Venugopal, S.; Kang, G.; Sarkar, R.; Ajjampur, S. Geographical distribution of soil transmitted helminths and the effects of community type in South Asia and South East Asia-A systematic review. PLoS Negl. Trop. Dis. 2018, 12, e6153. [CrossRef] [PubMed] 
16. Murray, P.R.; Rosenthal, K.S.; Pfaller, M.A. Medical Microbiology, 8th ed.; Elsevier: Philadelphia, PA, USA, 2015; pp. 748-751, ISBN 978-032-329-956-5.

17. Fung, I.C.; Cairncross, S. Ascariasis and handwashing. Trans. R. Soc. Trop. Med. Hyg. 2009, 103, $215-222$. [CrossRef] [PubMed]

18. Dold, C.; Holland, C.V. Ascaris and ascariasis. Microbes Infect. 2011, 13, 632-637. [CrossRef] [PubMed]

19. Coordinating Office of the National Survey on the Important Human Parasitic Diseases. A national Survey on Current Status of the Important Parasitic Diseases in Human Population. Chin. J. Parasitol. Parasit. Dis. 2005, 23, 332-340.

20. Bouzid, M.; Kintz, E.; Hunter, P.R. Risk factors for Cryptosporidium infection in low and middle income countries: A systematic review and meta-analysis. PLoS Negl. Trop. Dis. 2018, 12, e6553. [CrossRef] [PubMed]

21. Lv, S.; Tian, L.; Liu, Q.; Qian, M.; Fu, Q.; Steinmann, P.; Chen, J.; Yang, G.; Yang, K.; Zhou, X. Water-Related Parasitic Diseases in China. Int. J. Environ. Res. Public Health 2013, 10, 1977-2016. [CrossRef] [PubMed]

22. WHO. Soil-transmitted helminthiasis. Number of children treated 2007-2008: Update on the 2010 global target. Wkly. Epidemiol. Rec. 2010, 85, 141-147.

23. Moser, W.; Schindler, C.; Keiser, J. Efficacy of recommended drugs against soil transmitted helminths: Systematic review and network meta-analysis. BMJ 2017, 358, j4307. [CrossRef] [PubMed]

24. Menzies, S.K.; Rodriguez, A.; Chico, M.; Sandoval, C.; Broncano, N.; Guadalupe, I.; Cooper, P.J. Risk factors for soil-transmitted helminth infections during the first 3 years of life in the tropics; findings from a birth cohort. PLoS Negl. Trop. Dis. 2014, 8, e2718. [CrossRef] [PubMed]

25. WHO. Lack of Sanitation for 2.4 Billion People Is Undermining Health Improvements. Available online: http:/ / www.who.int/news-room/detail/30-06-2015-lack-of-sanitation-for-2-4-billion-people-isundermining-health-improvements (accessed on 31 July 2018).

26. Matthys, B.; Bobieva, M.; Karimova, G.; Mengliboeva, Z.; Jean-Richard, V.; Hoimnazarova, M.; Kurbonova, M.; Lohourignon, L.K.; Utzinger, J.; Wyss, K. Prevalence and risk factors of helminths and intestinal protozoa infections among children from primary schools in western Tajikistan. Parasit. Vector 2011, 4. [CrossRef] [PubMed]

27. Strunz, E.C.; Addiss, D.G.; Stocks, M.E.; Ogden, S.; Utzinger, J.; Freeman, M.C. Water, Sanitation, Hygiene, and Soil-Transmitted Helminth Infection: A Systematic Review and Meta-Analysis. PLoS Med. 2014, 11. [CrossRef] [PubMed]

28. Ziegelbauer, K.; Speich, B.; Maeusezahl, D.; Bos, R.; Keiser, J.; Utzinger, J. Effect of Sanitation on Soil-Transmitted Helminth Infection: Systematic Review and Meta-Analysis. PLoS Med. 2012, 9. [CrossRef] [PubMed]

29. Fuhrimann, S.; Winkler, M.S.; Kabatereine, N.B.; Tukahebwa, E.M.; Halage, A.A.; Rutebemberwa, E.; Medlicott, K.; Schindler, C.; Utzinger, J.; Cisse, G. Risk of Intestinal Parasitic Infections in People with Different Exposures to Wastewater and Fecal Sludge in Kampala, Uganda: A Cross-Sectional Study. PLoS Negl. Trop. Dis. 2016, 10, e4469. [CrossRef] [PubMed]

30. Cheng, S.; Li, Z.; Uddin, S.; Mang, H.P.; Zhou, X.; Zhang, J.; Zheng, L.; Zhang, L. Toilet revolution in China. J. Environ. Manag. 2018, 216, 347-356. [CrossRef] [PubMed]

31. Johansen, A.; Nielsen, H.B.; Hansen, C.M.; Andreasen, C.; Carlsgart, J.; Hauggard-Nielsen, H.; Roepstorff, A. Survival of weed seeds and animal parasites as affected by anaerobic digestion at meso- and thermophilic conditions. Waste Manag. 2013, 33, 807-812. [CrossRef] [PubMed]

32. Bowen, A.; Ma, H.; Ou, J.; Billhimer, W.; Long, T.; Mintz, E.; Hoekstra, R.M.; Luby, S. A cluster-randomized controlled trial evaluating the effect of a handwashing-promotion program in Chinese primary schools. Am. J. Trop. Med. Hyg. 2007, 76, 1166-1173. [PubMed]

33. Yang, G.J.; Liu, L.; Zhu, H.R.; Griffiths, S.M.; Tanner, M.; Bergquist, R.; Utzinger, J.; Zhou, X.N. China's sustained drive to eliminate neglected tropical diseases. Lancet Infect. Dis. 2014, 14, 881-892. [CrossRef]

34. WHO. Working to Overcome the Global Impact of Neglected Tropical Diseases: First WHO Report on Neglected Tropical Diseases. Available online: http://apps.who.int/iris/handle/10665/44440 (accessed on 28 July 2018).

35. Freeman, M.C.; Ogden, S.; Jacobson, J.; Abbott, D.; Addiss, D.G.; Amnie, A.G.; Beckwith, C.; Cairncross, S.; Callejas, R.; Colford, J.J.; et al. Integration of water, sanitation, and hygiene for the prevention and control of neglected tropical diseases: A rationale for inter-sectoral collaboration. PLoS Negl. Trop. Dis. 2013, 7, e2439. [CrossRef] [PubMed] 
36. Abeywardena, H.; Jex, A.R.; Gasser, R.B. A perspective on Cryptosporidium and Giardia, with an emphasis on bovines and recent epidemiological findings. Adv. Parasitol. 2015, 88, 243-301. [CrossRef] [PubMed]

37. Yang, Y.; Zhou, Y.; Cheng, W.; Pan, X.; Xiao, P.; Shi, Y.; Gao, J.; Song, X.; Chen, Y.; Jiang, Q. Prevalence and Determinants of Cryptosporidium Infection in an Underdeveloped Rural Region of Southwestern China. Am. J. Trop. Med. Hyg. 2017, 96, 595-601. [CrossRef] [PubMed]

38. Hofstra, N.; Vermeulen, L.C. Impacts of population growth, urbanisation and sanitation changes on global human Cryptosporidium emissions to surface water. Int. J. Hyg. Environ. Health 2016, 219, 599-605. [CrossRef] [PubMed]

39. Jasper, C.; Le, T.T.; Bartram, J. Water and sanitation in schools: A systematic review of the health and educational outcomes. Int. J. Environ. Res. Public Health 2012, 9, 2772-2787. [CrossRef] [PubMed]

40. Herbst, S.; Fayzieva, D.; Kistemann, T. Risk factor analysis of diarrhoeal diseases in the Aral Sea area (Khorezm, Uzbekistan). Int. J. Environ. Health Res. 2008, 18, 305-321. [CrossRef] [PubMed]

(C) 2018 by the authors. Licensee MDPI, Basel, Switzerland. This article is an open access article distributed under the terms and conditions of the Creative Commons Attribution (CC BY) license (http://creativecommons.org/licenses/by/4.0/). 